Drug Anal Res, 2018; 02, 27-36

\title{
Evaluation of the presence of polymorphic forms and influence on the dissolution profile of Tenoxicam in active pharmaceutical ingredient and formulations
}

\author{
Aline Taís Fries ${ }^{a^{*}}$, Natália Olegário ${ }^{a}$, Sarah Chagas Campanharo ${ }^{a}$, Vitor Paulo Pereira ${ }^{b}$, and Martin Steppe ${ }^{a}$ \\ a Programa de Pós-Graduação em Ciências Farmacêuticas, Faculdade de Farmácia, Universidade Federal do Rio Grande do Sul \\ (UFRGS), Porto Alegre - RS, Brazil \\ ${ }^{b}$ Programa de Pós-Graduação em Geociências, Faculdade de Geociências, Universidade Federal do Rio Grande do Sul \\ (UFRGS), Porto Alegre-RS, Brazil
}

\section{*Corresponding author: farmaceuticaf@yahoo.com}

Polymorphism is a relatively common phenomenon among pharmaceutical compounds, and one of the main aspects to be considered in the production and development of medications. The investigation of polymorphism associated with oxicams, a group belonging to the class of non-steroidal anti-inflammatory drugs (NSAIDs) has increased in recent years and, in the case of tenoxicam, the existence of four polymorphic forms is reported in the literature. The objective of this study was to characterize the presence of different polymorphic forms of tenoxicam in active pharmaceutical ingredient and oral pharmaceutical formulations, as well as to evaluate the influence on in vitro dissolution. The characterization of the three samples of pharmaceutical ingredient of tenoxicam from different suppliers by X-Ray Diffraction (XRD), Infrared (IR) and dissolution profile indicated the presence of a form III crystalline structure, without presenting significant differences between the in vitro dissolution profiles analyzed, and a Dissolution Efficiency (DE\%) of $60.30 \%, 60.70 \%$ and $72.34 \%$, respectively. When the four pharmaceutical specialties of tenoxicam were submitted to XRD analysis, they also presented form III crystalline structures. Despite this, the formulations presented different dissolution profiles and a DE\% of $75.23 \%, 83.69 \%, 78.19 \%$ and $90.63 \%$, respectively, without compromising their quality. However, often polymorphism affects physico-chemical properties of drugs, showing the importance of studying this phenomenon, by correlating the presence of crystalline structures to alterations in the quality of active ingredients and pharmaceutical products.

Keywords: tenoxicam, polymorphism, crystalline structure, active pharmaceutical ingredients, pharmaceutical formulations.

\section{Introduction}

Polymorphism is a frequent phenomenon among pharmaceutical compounds, and one of the main aspects to be considered in the production and development of medications. Its presence may impair the health of the population and have a strong economic impact on the pharmaceutical industries. According to the polymorph involved in the production, the medication may present as less active, active or toxic to the human organism $(1,2)$. It is decisively important to understand the relationship between crystalline material and its properties in order to select the most appropriate polymorph for the development of a pharmaceutical specialty. Polymorphism may directly influence the mechanical properties of active pharmaceutical ingredient such as flux, elasticity, compressibility and force of bonding, and also the bioavailability of the pharmaceutical product $(3,4)$. Moreover, changes may occur in the crystalline structure resulting from the industrial production process, reinforcing the importance of studying polymorphic behavior involving finished products $(5,6)$.

Transformations induced by processing during the production of pharmaceutical products are difficult to predict and control. Subjecting an active pharmaceutical ingredient with a given crystalline structure to industrial processing may induce modifications in the crystalline phase. The milling process may generate significant amounts of heat, consequently generating transformations of the crystalline phase and amorphous components. Wet granulation also provides an ideal environment for the crystallization of new crystalline phases, as it involves the mixture of the active ingredient and excipients with a granulation liquid, and also requires the product to be dried and milled $(7,8)$.

Polymorphism may affect various pharmaceutical classes including the oxicam group. This group belongs to the class of Non-Steroidal Anti-inflammatory Drugs (NSAIDs) (9). The investigation of polymorphism associated with oxicams has increased in recent years (10), and data from the literature report the existence of four polymorphic forms (forms I, II, III and IV) for tenoxicam (11). It is therefore relevant to perform tests that will characterize the solid state of this drug aiming to analyze the quality parameters of active pharmaceutical ingredient and finished products, since the possibility of polymorphism is a critical factor for the pharmaceutical industries. Therefore, the objective of this study was to evaluate the crystalline structure and influence on the in vitro dissolution profile of tenoxicam in active pharmaceutical ingredient and oral pharmaceutical formulations available in the consumer market. 
Drug Anal Res, 2018; 02, 27-36

\section{Material and Methods}

\section{Reference standard}

The standard used was compound 4-hydroxy-2-methyl-N-2pyrimidilo-2H-thiene [2,3-e] -1,2-thiazine-3-carboxamide1,1-dioxide (tenoxicam) obtained from Changzhou Hubin Medicine Raw Materials Co. Ltd (Changzhou, China), with a declared content of $99.59 \%$.

\section{Samples}

Three samples of active pharmaceutical ingredient of tenoxicam (GTE1, STE2 and NTE3) evaluated were acquired from the following pharmacochemical industries: Glenmark Generics Ltd. (Daund, India), Sifovitor Infa Group S.p.A. (Casaletto Lodigiano, Italy) and Nantong Jinhua Pharmaceutical Co. Ltd (Nantong, China), respectively.

Samples of pharmaceutical specialties (VTE0, RTE1, ETE2 and MTE3) containing tenoxicam at a concentration of 20 mg were obtained from Brazilian pharmaceutical industries. The placebos were prepared in the laboratory based on the composition of the excipients present in the samples of tablets, namely, lactose, amide, talcum, magnesium stearate, sodium amidoglycolate, hypromellose, sodium croscarmellose, microcrystalline cellulose, hydroxypropylmethylcellulose, ethylcellulose, macrogol, polyethylenoglycol, titanium dioxide, silicon dioxide, tartrazine yellow, and yellow iron oxide. Monobasic Potassium Phosphate $\left(\mathrm{KH}_{2} \mathrm{PO}_{4}\right)$ was obtained from Merck (Darmstadt, Germany).

\section{Tenoxican polymorphs}

Tenoxicam polymorphs used were prepared according to the procedures described in the literature (11) and the conditions selected were as follows:

Preparation of form I: Tenoxicam was dissolved in chloroform (Nuclear ${ }^{\circledR}$, Lot 09111520$)$ at $60{ }^{\circ} \mathrm{C}(30 \mathrm{mg} / \mathrm{ml})$ and then cooled at a temperature of $4{ }^{\circ} \mathrm{C}$ (ice bath). After six days stored at room temperature it was filtrated.

Preparation of form II: Tenoxicam was dissolved in butanol (Labsynth ${ }^{\circledR}$, Lot 114005) at room temperature (3 $\mathrm{mg} / \mathrm{ml}$ ) and kept in this condition for two days. After the stipulated time, filtration was performed.

Preparation of form III: Tenoxicam was dissolved in isopropylic alcohol (Tedia ${ }^{\circledR}$, Lot 903139) at room temperature $(2 \mathrm{mg} / \mathrm{ml})$, it was cooled at $4{ }^{\circ} \mathrm{C}$ (ice bath) and the content was isolated by filtration.

Preparation of form IV: Tenoxicam was dissolved in methanol (Merck ${ }^{\circledR}$, Lot 1672807) at room temperature (2 $\mathrm{mg} / \mathrm{ml}$ ) and left to rest in this state for two days. Filtration was performed after the stipulated time.

\section{Thermal Analyses}

A Shimadzu ${ }^{\circledR}$ Differential Scanning Calorimeter (Kyoto, Japan), model DSC-60 with heat flux and calibrated with indium $\left(156.6{ }^{\circ} \mathrm{C}\right.$, transition energy $\left.-28.45 \mathrm{~J} / \mathrm{g}^{-1}\right)$ and zinc $\left(419.58{ }^{\circ} \mathrm{C}\right.$, transition energy $\left.-100.50 \mathrm{~J} / \mathrm{g}^{-1}\right)$ was used. It was equipped with a FC-60A flux controller, TA $60 \mathrm{WS}$ integrator and control and analysis software TA 60 version 2.0 .

The DSC curves were obtained in an inert nitrogen atmosphere with a $50 \mathrm{ml} / \mathrm{min}$ flow and heating rate of $10^{\circ} \mathrm{C} /$ min beginning at $25^{\circ} \mathrm{C}$ to $250^{\circ} \mathrm{C}$. Recrystallized samples of tenoxicam (forms I, II, III and IV) ranging from 1.5 to 1.95 mg were placed on hermetic aluminium pans.

\section{X-Ray Diffraction}

The Siemens ${ }^{\circledR}$ diffractometer, model D-5000 (Munich, Germany) was used to analyze the samples. It was equipped with Difrac ${ }^{\circledR}$ Plus EVA version 11.0 to identify and index the peaks by using comparison algorithms based on the JCPDS (Joint Committee on Power Diffraction Standards) database. The analysis were performed at room temperature, with samples in powder form, about 1 gram, using $\lambda \mathrm{Cu}_{\mathrm{K} \alpha}$ radiation (1.5409 $\AA$ ), that were scanned between 2 and $72^{\circ}$ $(2 \theta)$, with speed of $0.02 \% 1 \mathrm{~s}$, using an energy of $40 \mathrm{kV}$ and $25 \mathrm{~mA}$. The tablets submitted to the test were milled in an agate mortar.

The technique was performed to look at the crystalline structure present in the standard tenoxicam, in the crystalline forms prepared (forms I, II, III and IV), in the active pharmaceutical ingredient (GTE1, STE2 and NTE3) and in the pharmaceutical tablets of the drug (VTE0, RTE1, ETE2 and MTE3).

\section{Spectrophotometry by IV}

The absorption spectrum of the IV region was obtained using a Perkin Elmer ${ }^{\circledR}$ model FT-IR Spectrum BX Spectrophotometer (Massachusetts, USA) in the 600 to 4000 $\mathrm{cm}^{-1}$ range with a scanning number of 100 scans. Approximately $1 \mathrm{mg}$ of powders from each sample was used for analysis.

The technique was used to identify standard tenoxicam, evaluate the forms I, II, III and IV prepared, and to characterize the active pharmaceutical ingredient of tenoxicam (GTE1, STE2 and NTE3).

\section{Dissolution Profile}

The procedure involved the use of the Varian ${ }^{\circledR}$ dissolution apparatus (California, USA) model VK 7010 equipped with 6 vessels, multiple automatic sampler controlled by an automatic station model VK 8000 , bidirectional peristaltic pump, model VK, and digital heater/circulator model VK 750D. The experimental conditions were according to those favored by the British Pharmacopoeia (12). To perform the test apparatus 2 (paddles) were used with an agitation velocity of $50 \mathrm{rpm}$, vessels containing $900 \mathrm{ml}$ of monobasic potassium phosphate $\mathrm{pH} 6.8$ as a dissolution medium at a temperature of $37{ }^{\circ} \mathrm{C} \pm 0.5^{\circ} \mathrm{C}$.

In order to evaluate the in vitro dissolution profile of the tenoxicam tablets (VTE0, RTE1, ETE2 and MTE3), $10 \mathrm{ml}$ aliquots were removed from each of the vessels, adequately filtered with $0.45 \mu \mathrm{m}$ filters at times of $2,5,10,20$ and 25 minutes. For the active pharmaceutical ingredients (GTE1, 
STE2 and NTE3) evaluated, $20.0 \mathrm{mg}$ of sample were weighed and $10 \mathrm{ml}$ aliquots were removed from each of the vessels, adequately, filtered with $0.45 \mu \mathrm{m}$ filters at times of $5,10,15,20,25,30,35,40,45$ and 50 minutes.

The absorption spectrum in the UV region was obtained using a Shimadzu ${ }^{\circledR}$ spectrophotometer (Kyoto, Japan), model UV-1800, equipped with quartz cuvettes, with an optical path of $1.0 \mathrm{~cm}$ and UV Probe software, version 2.33 to process the data. The amount of drug released into the dissolution medium was determined at a wavelength of 368 $\mathrm{nm}$.

The results of the dissolution profiles were evaluated by the independent model method, calculating factors $f 1$ and $f 2$, and by the dependent model method using mathematical equations referring to zero order and first order. Dependent models were applied using Micromath Scientist for Windows software Version 2.01. Other parameters used to characterize the dissolution profile were the half life time $\left(t_{50 \%}\right)$, amount released from the drug in 25 minutes $\left(\mathrm{Q}_{20}\right)$ and dissolution efficiency (DE\%).

\section{Results and discussion}

\section{Differential Scanning Calorimetry (DSC)}

\section{Tenoxicam polymorphs preparations}

The DSC curves obtained for different recrystallization conditions of tenoxicam did not present significant changes compared to the DSC data expected for the drug (Figure 1), which presents an endothermic peak (fusion) close to 215.41 ${ }^{\circ} \mathrm{C}$, followed by a an exothermic peak (degradation) (13).



Figure 1. Thermal profile of recrystallized samples of tenoxicam in a nitrogen atmosphere of $50 \mathrm{ml} / \mathrm{min}$ with a heating rate of 10 ${ }^{\circ} \mathrm{C} / \mathrm{min}$, using a Shimadzu ${ }^{\circledR}$ Differential Scanning Calorimeter model DSC-60.

Thus, no atypical event was observed in the samples (Figure 3), because they all presented an endothermic event (heat absorption), close to the original fusion range of tenoxicam located between $209^{\circ} \mathrm{C}$ and $213^{\circ} \mathrm{C}$ (14).

When the polymorphism is present, the DSC curves have a number of fusion peaks characteristic of the crystalline forms present, and also, thermal events such as decomposition and recrystallization are often observed when DSC is used to characterize polymorphs $(6,4)$.
A single tenoxicam polymorphic form obtained (form III) is related to its molecular shape, considering its low flexibility (a factor which reduces the tendency to crystallization) and the chemical bonds present in the molecule (Van der Waals Forces, Dipole-Dipole and Hydrogen Bridges), that directly influence the recrystallization process. The intermolecular forces influence the molecular force and orientation, while conformational flexibility interferes both in the way the molecules are packed inside the crystals and in the spatial orientation of the molecule $(15,16)$.

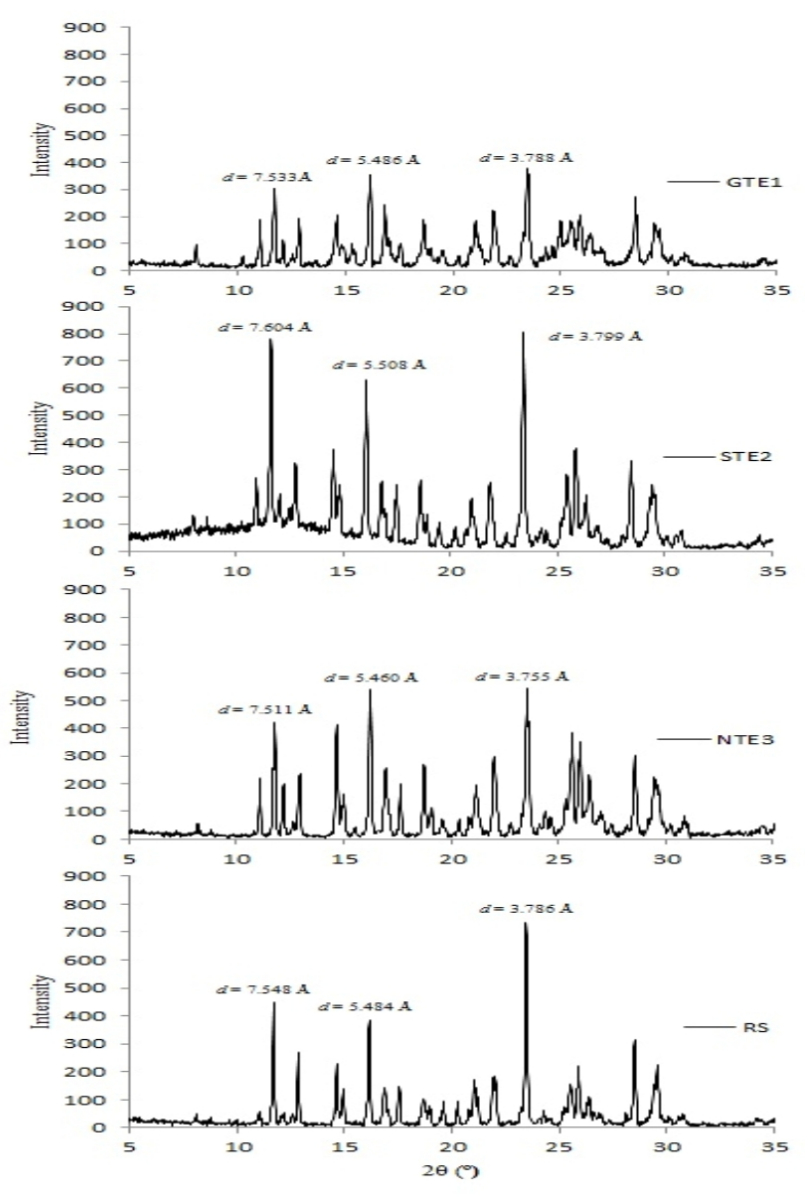

Figure 3. Comparison between the diffractograms of active pharmaceutical ingredients of tenoxicam and RS, obtained using the Siemens ${ }^{\circledR}$ Diffractometer, model D-5000, with $\lambda \mathrm{Cu}_{\mathrm{K} \alpha}$ radiation $(1.5409 \AA)$ and power of $40 \mathrm{kV}$ and $25 \mathrm{~mA}$.

\section{X-Ray Diffraction}

\section{Tenoxicam polymorphs preparations}

The crystalline structure of the standard tenoxicam (Changzhou Hubin Medicine Raw Materials Co. Ltd, Changzhou, China) presents a form III crystallographic structure according to data from the literature $(11,17)$. Different conditions for obtaining polymorphic forms of tenoxicam (forms I, II, III and IV) were applied according to Cantera et al. (2002) (11). However the different preparation processes of tenoxicam polymorphs did not lead to modifications in the original crystalline structure of this drug. The main peaks in the standard tenoxicam diffractogram at $8.12^{\circ} ; 10.98^{\circ} ; 11.04^{\circ} ; 12.86^{\circ} ; 14.64^{\circ}$; 
$14.96^{\circ} ; 16.16^{\circ} ; 16.84^{\circ} ; 16.88^{\circ} ; 17.56^{\circ} ; 18.7^{\circ} ; 19.62^{\circ} ;$ $21.94^{\circ} ; 23.46^{\circ} ; 24.3^{\circ} ; 25.54^{\circ} ; 28.52^{\circ} ; 28.52^{\circ} ; 29.58^{\circ}$ and $30.74^{\circ} 2 \theta$ presented positions similar to the peaks found in the diffractograms of forms I, II, III and IV, with close interplanar distances (values of $d$ ) between the three main peaks (Figure 2). Therefore, the addition of different solvent in samples of standard tenoxicam under the conditions proposed by Cantera, Leza and Bachiller (2002) (11), did not promote significant changes in the crystalline structure (form III). This information was also corroborated by the results previous obtained by Bolla et. al. (2013) (18) and Patel et. al. (2012) (17) who, based on the methodologies of Cantera et. al. (2002) (11), also only obtained form III.



Figure 2. Comparison of the diffractograms of the crystalline structures forms I, II, III and IV to tenoxicam RS, using a Siemens ${ }^{\circledR}$ Diffractometer model D-5000, with a $\lambda \mathrm{Cu}_{\mathrm{K} \alpha}$ radiation $(1.5409 \AA)$ and power of $40 \mathrm{kV}$ and $25 \mathrm{~mA}$.

\section{Analysis of the active pharmaceutical ingredients of tenoxicam}

The peaks present in the active ingredient GTE1, STE2 and NTE3 evaluated present positions similar to those presented by standard tenoxicam at $8.12^{\circ} ; 10.98^{\circ} ; 11.04^{\circ} ; 12.86^{\circ}$; $14.64^{\circ} ; 14.96^{\circ} ; 16.16^{\circ} ; 16.84^{\circ} ; 16.88^{\circ} ; 17.56^{\circ} ; 18.7^{\circ}$; $19.62^{\circ} ; 21.94^{\circ} ; 23.46^{\circ} ; 24.3^{\circ} ; 25.54^{\circ} ; 28.52^{\circ} ; 28.52^{\circ} ; 29.58^{\circ}$ and $30.742 \theta$ (Figure 3 ), configuring the same crystalline form. Factors such as nature and concentration of the solvent, cooling velocity, time and temperature involved in the synthesis of an active ingredient may influence the formation of distinct crystalline structures (19).

Therefore, although they are of completely different origins, the active pharmaceutical ingredients of tenoxicam (GTE1, STE2 and NTE3) from different pharmaceutical industries presented a crystalline structure similar to the standard tenoxicam (form III).

\section{Analysis of the tenoxicam tablets}

Figure 4 shows the evaluation of the simulated samples of the tenoxicam tablets by XRD. The diffractograms of the simulated samples of pharmaceutical products VTE0, RT1, ETE2 and MTE3 are found to present the three most intense peaks with very close $2 \theta$ positions (the arrows indicate the highest intensity peaks).

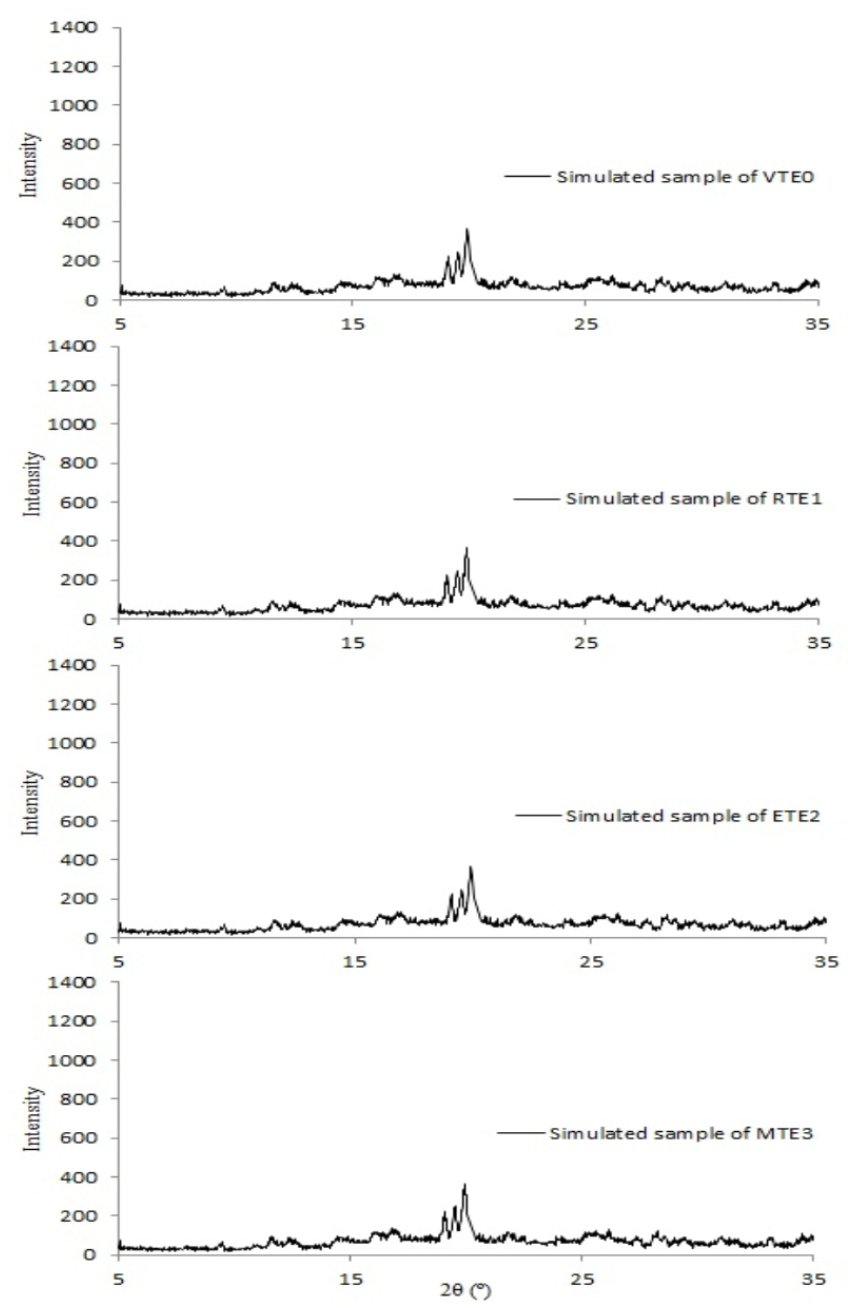

Figure 4. Comparison between the diffractograms of simulated samples of the tenoxicam tablets obtained using the Siemens ${ }^{\circledR}$ Diffractometer, model D-5000, with $\lambda \mathrm{Cu}_{\mathrm{K} \alpha}$ radiation (1.5409 $\AA$ ) and power of $40 \mathrm{kV}$ and $25 \mathrm{~mA}$.

When excluding the peaks corresponding to the excipients from the evaluation, and evaluating those that correspond to the drug tenoxicam (Figure 5), it is seen that there are modifications in the intensities and small alterations in the position of the peaks of crystalline structures belonging to 
the compounds VTE0, RT1, ETE2 and MTE3 in relation to the peaks of standard tenoxicam positioned at $8.12^{\circ} ; 10.98^{\circ}$; $11.04^{\circ} ; 12.86^{\circ} ; 14.64^{\circ} ; 14.96^{\circ} ; 16.16^{\circ} ; 16.84^{\circ} ; 16.88^{\circ}$; $17.56^{\circ} ; 18.7^{\circ} ; 19.62^{\circ} ; 21.94^{\circ} ; 23.46^{\circ} ; 24.3^{\circ} ; 25.54^{\circ} ; 28.52^{\circ}$; $28.52^{\circ} ; 29.58^{\circ}$ and $30.74^{\circ} 2 \theta$.

The shifts in the positions of the peaks (20) in the of tenoxicam tablets (Figure 5) are possibly the result of transformations induced by processing (PIT) (7). Although the industrial processes may cause changes in the crystalline structure of pharmaceutical compounds (8), these procedures were unable to form the new crystalline phase in tenoxicam, present in the pharmaceutical specialties evaluated, since no formation of new peaks or subtraction of those characteristic of the drug is observed, maintaining the form III structures. In this way, the crystalline structure of tenoxicam, form III, proved to be stable during the drug production process. A similar result was evidenced by the XRD technique for risperidone which maintained its crystalline structure (polymorph A) without modifications after undergoing industrial processing (20).

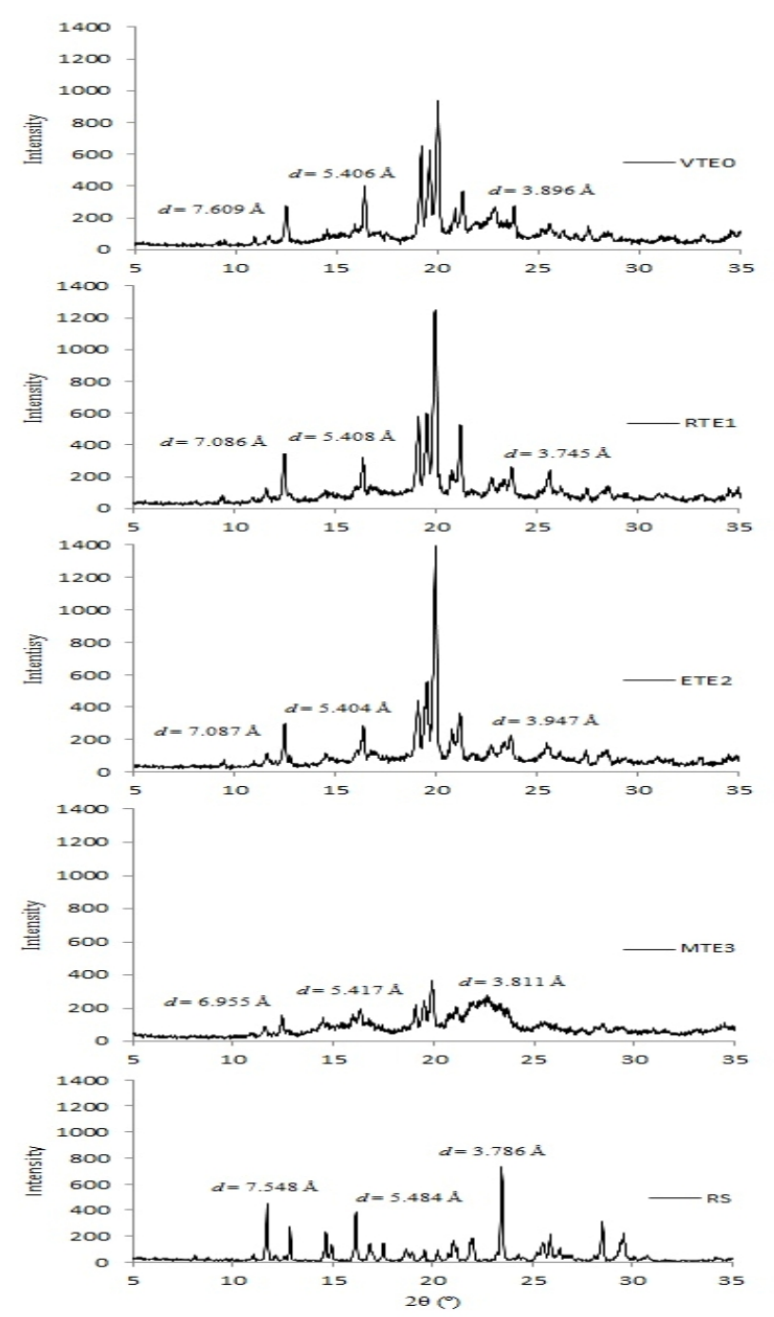

Figure 5. Comparison between the diffractograms of the pharmaceutical specialties and RS obtained using the Siemens ${ }^{\circledR}$ Diffractometer, model D-5000, with $\lambda \mathrm{Cu}_{\mathrm{K} \alpha}$ radiation $(1.5409 \AA$ ) and power of $40 \mathrm{kV}$ and $25 \mathrm{~mA}$.

\section{IR Spectrophotometry}

\section{Tenoxicam polymorphs preparations}

The absorption frequencies of the main IR bands and their respective attributions for tenoxicam are described in Table 1 (21). Considering the absorption frequencies of the main bands of tenoxicam in IR (Table 1) and the spectra of the crystalline structures (form I, II, III and IV), and standard tenoxicam (Figure 6), there is a similarity between the spectra with an absence of alterations on the IR bands. The polymorphism causes shifts in the characteristic bands of the substances resulting from the different intermolecular interactions of each crystalline structure (6). This does not occur with the samples prepared according to the methodology of Cantera, Leza and Bachiller (2002) (11).

Table 1. Absorption frequencies of the main IR bands and their respective attributions for tenoxicam.

\begin{tabular}{lcc}
\hline \multicolumn{1}{c}{ ATTRIBUTION } & $\begin{array}{c}\text { ABSORPTION } \\
\text { BAND } \\
\left(\mathbf{c m}^{-1}\right)\end{array}$ & $\begin{array}{c}\text { OBSERVED } \\
\left(\mathbf{c m}^{-1}\right)\end{array}$ \\
\hline $\begin{array}{l}\text { Axial deformation } \\
\text { C-H aromatics }\end{array}$ & $3100-3000$ & 3064 \\
$\begin{array}{l}\text { Vibration of axial } \\
\text { deformation of C=O } \\
\text { in secondary amides }\end{array}$ & 1640 & 1634 \\
$\begin{array}{l}\text { Weak bands of } \\
\text { combination and }\end{array}$ & $2000-1600$ & \\
harmonics between & & 1594 \\
$\begin{array}{l}\text { C-H } \\
\text { Vibration of angular } \\
\text { deformation of N-H } \\
\text { in secondary amides }\end{array}$ & $1570-1515$ & \\
$\begin{array}{l}\text { Vibration of angular } \\
\text { deformation on } \\
\text { plane O-H }\end{array}$ & $1420-1330$ & 1555 \\
$\begin{array}{l}\text { Vibration of axial } \\
\text { deformation of C-O } \\
\text { in alcohols }\end{array}$ & $1200-1000$ & 1382 \\
$\begin{array}{l}\text { Axial deformation of } \\
\text { C-O of secondary } \\
\text { alcohols, with } \\
\text { alicyclic ring of five } \\
\text { or six atoms }\end{array}$ & $1085-1050$ & 1322 \\
\hline
\end{tabular}

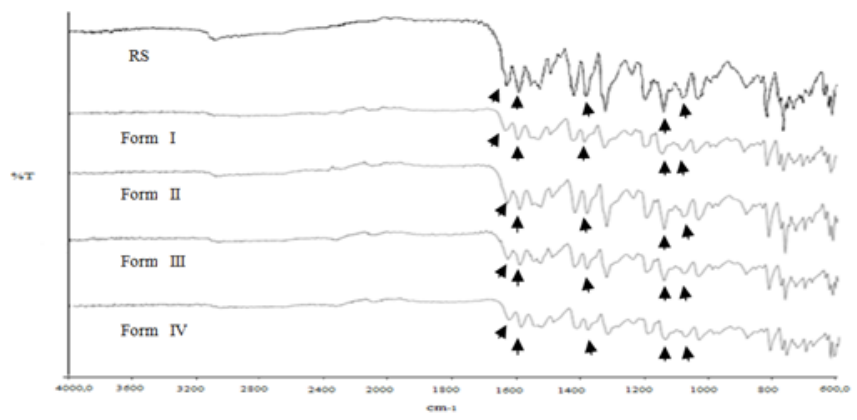

Figure 6. Spectrum in the IR (600 to $4000 \mathrm{~cm}^{-1}$ ) of the crystalline forms of tenoxicam obtained using the Perkin Elmer $^{\circledR}$ Spectrophotometer model FT-IR Spectrum BX, with an accessory for attenuated total reflectance (ATR). 
Analysis of the active pharmaceutical ingredients of tenoxicam

Figure 7 records the IR spectrum of the active pharmaceutical ingredients of tenoxicam, where a similar spectral profile is seen between the active pharmaceutical ingredients of tenoxicam called GTE1, STE2 and NTE3 and standard tenoxicam. No shifts are seen in the characteristic bands, a peculiarity of polymorphism (6), since the absorption bands expected for the tenoxicam molecule are present in the samples, with bands at $1,634 \mathrm{~cm}^{-1}$ (vibration of axial deformation of $\mathrm{C}=\mathrm{O}$ in secondary amides), 1,594 $\mathrm{cm}^{-1}$ (weak bands of combination and harmonics between $\mathrm{C}$ $\mathrm{H}), 1,382 \mathrm{~cm}^{-1}$ (vibration of angular deformation on plane O$\mathrm{H}$ ), $1,146 \mathrm{~cm}^{-1}$ (vibration of axial deformation of $\mathrm{C}-\mathrm{O}$ in alcohols) and $1,084 \mathrm{~cm}^{-1}$ (axial deformation of $\mathrm{C}-\mathrm{O}$ of secondary alcohols, with an alicicyclic ring of five or six atoms) (21).

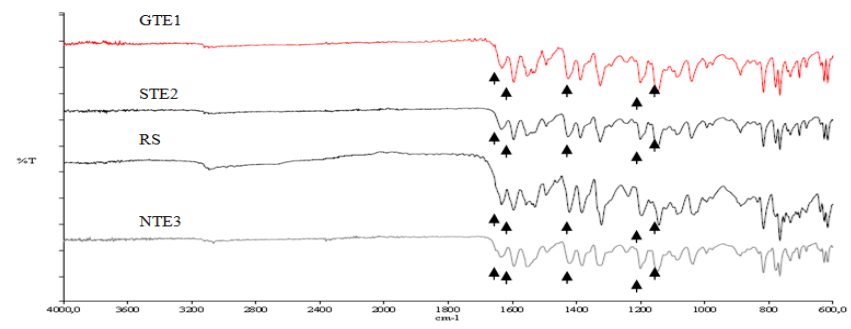

Figure 7. IR spectrum IV (600 to $\left.4000 \mathrm{~cm}^{-1}\right)$ for the pharmaceutical ingredients of tenoxicam obtained using a Perkin Elmer $^{\circledR}$ Spectrophotometer model FT-IR Spectrum BX with an accessory for attenuated total reflectance (ATR).

\section{Dissolution Profile}

Analysis of the active pharmaceutical ingredients of tenoxicam

Considering that solid substances may undergo changes in their crystalline states due to the compaction to which they are submitted, it was decided to dissolve the active ingredients in powder form instead of intrinsic dissolution, which requires compression of the solid material to be analyzed. The modification of the solid form of the sample due to the compression used would invalidate the intrinsic dissolution assay (3). Table 2 and Figure 8 show the results obtained in the dissolution profiles of pharmaceutical ingredients GTE1, STE2 and NTE3 in powder form. In the dissolution profiles it is seen that within 30 minutes all the active pharmaceutical ingredients GTE1, STE2 and NTE3 are dissolved, with about $75 \%$ dissolution, and no significant difference between the dissolution profiles evaluated.

Table 2. Mean results obtained studying the particulate dissolution profile of the pharmaceutical ingredients of tenoxicam.

\begin{tabular}{ccccccc}
\hline TIME & \multicolumn{2}{c}{ GTE1 } & \multicolumn{2}{c}{ STE2 } & \multicolumn{2}{c}{ NTE3 } \\
\cline { 2 - 7 }$(\mathbf{m i n})$ & $\begin{array}{c}\text { \% dissolved } \\
\pm \text { standard deviation }\end{array}$ & $\begin{array}{c}\text { RSD } \\
(\%)\end{array}$ & $\begin{array}{c}\text { \% dissolved } \pm \\
\text { standard deviation }\end{array}$ & $\begin{array}{c}\text { RSD } \\
(\%)\end{array}$ & $\begin{array}{c}\text { \% dissolved } \\
\pm \text { standard } \\
\text { deviation }\end{array}$ & $\begin{array}{c}\text { RSD } \\
(\%)\end{array}$ \\
\hline 5 & $19.22 \pm 0.78$ & 8.91 & $21.74 \pm 0.83$ & 9.30 & $37.29 \pm 0.70$ & 8.10 \\
10 & $39.07 \pm 0.44$ & 3.65 & $39.93 \pm 0.56$ & 5.10 & $56.24 \pm 0.35$ & 2.83 \\
15 & $53.88 \pm 0.55$ & 4.60 & $54.44 \pm 0.34$ & 2.30 & $70.67 \pm 0.31$ & 2.10 \\
20 & $64.77 \pm 0.42$ & 3.21 & $64.15 \pm 0.43$ & 3.38 & $80.41 \pm 0.40$ & 3.00 \\
25 & $71.65 \pm 0.37$ & 2.92 & $70.65 \pm 0.48$ & 4.22 & $84.97 \pm 0.53$ & 4.40 \\
30 & $75.48 \pm 0.38$ & 3.00 & $75.27 \pm 0.32$ & 2.15 & $86.27 \pm 0.24$ & 1.20 \\
35 & $78.33 \pm 0.54$ & 4.42 & $78.28 \pm 0.45$ & 3.70 & $87.12 \pm 0.22$ & 1.18 \\
40 & $79.50 \pm 0.56$ & 5.30 & $79.89 \pm 0.30$ & 1.70 & $87.80 \pm 0.44$ & 3.52 \\
45 & $80.35 \pm 0.48$ & 4.20 & $81.56 \pm 0.46$ & 3.75 & $88.22 \pm 0.58$ & 5.79 \\
50 & $81.64 \pm 0.36$ & 2.87 & $82.11 \pm 0.47$ & 4.00 & $88.79 \pm 0.55$ & 4.75 \\
\hline
\end{tabular}




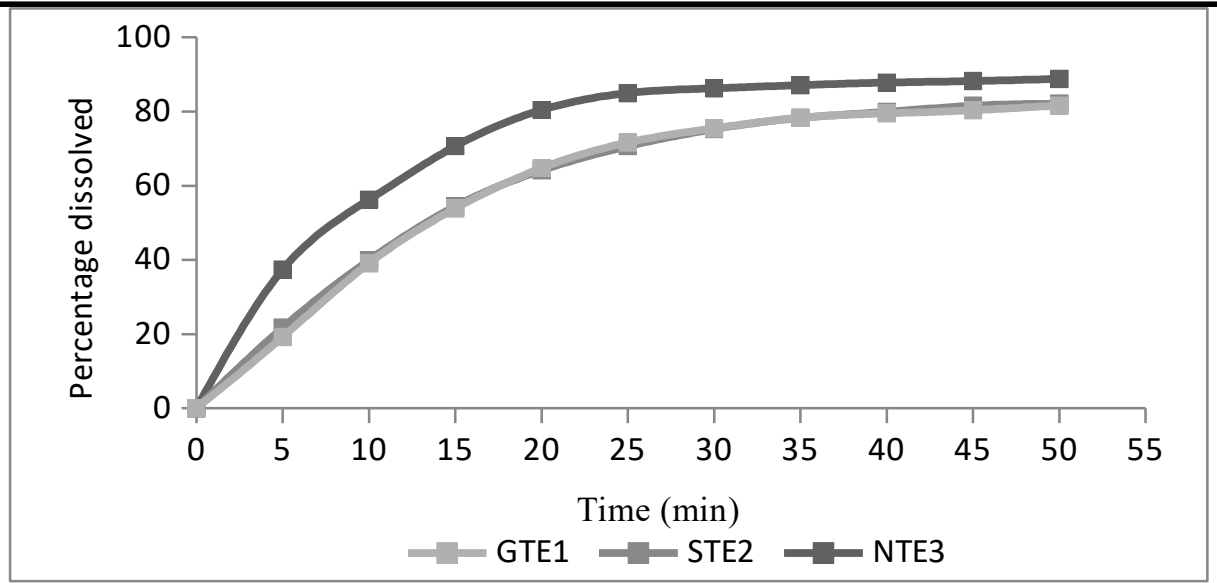

Figure 8. Graphic representation of the particulate dissolution of four tenoxicam raw material suppliers represented by the acronyms GTE1, STE2 and NTE3.

Table 3. Mean results obtained in the study of the dissolution profile of the tenoxicam tablets at different laboratories at times $2,5,10,15$, 20 and 25 minutes. ${ }^{\mathrm{a}}$

\begin{tabular}{ccccccccc}
\hline \multirow{2}{*}{ TIME } & \multicolumn{2}{c}{ VTE0 } & \multicolumn{2}{c}{ RTE1 } & \multicolumn{2}{c}{ ETE2 } & \multicolumn{2}{c}{ MTE3 } \\
\cline { 2 - 9 }$(\boldsymbol{m i n})$ & $\begin{array}{c}\text { \% dissolved } \\
\pm \text { standard } \\
\text { deviation }\end{array}$ & $\begin{array}{l}\text { RSD } \\
(\%)\end{array}$ & $\begin{array}{c}\text { \% dissolved } \\
\pm \text { standard } \\
\text { deviation }\end{array}$ & $\begin{array}{c}\text { RSD } \\
(\%)\end{array}$ & $\begin{array}{c}\text { \% dissolved } \\
\pm \text { standard } \\
\text { deviation }\end{array}$ & $\begin{array}{c}\text { RSD } \\
(\%)\end{array}$ & $\begin{array}{c}\text { \% dissolved } \pm \\
\text { standard deviation }\end{array}$ & $\begin{array}{c}\text { RSD } \\
(\%)\end{array}$ \\
\hline 2 & $37.47 \pm 0.97$ & 7.52 & $42.59 \pm 0.95$ & 7.13 & $43.83 \pm 1.41$ & 12.49 & $62.46 \pm 0.94$ & 6.95 \\
5 & $64.02 \pm 0.69$ & 3.88 & $72.98 \pm 0.73$ & 4.48 & $62.79 \pm 0.68$ & 3.61 & $91.61 \pm 0.65$ & 3.06 \\
10 & $79.15 \pm 0.73$ & 4.34 & $90.42 \pm 0.48$ & 2.22 & $77.08 \pm 0.26$ & 1.28 & $97.98 \pm 0.62$ & 2.94 \\
15 & $87.85 \pm 0.62$ & 2.91 & $97.10 \pm 0.48$ & 2.19 & $90.21 \pm 0.25$ & 1.25 & $99.43 \pm 0.56$ & 2.51 \\
20 & $92.56 \pm 0.61$ & 2.83 & $99.21 \pm 0.28$ & 1.38 & $100.46 \pm 0.31$ & 1.51 & $100.29 \pm 0.59$ & 2.65 \\
25 & $94.52 \pm 0.67$ & 3.55 & $101.12 \pm 0.25$ & 1.13 & $102.12 \pm 0.53$ & 2.31 & $101.82 \pm 0.57$ & 2.5 \\
\hline
\end{tabular}

${ }^{\mathrm{a}}$ Values represent the mean of 12 determinations.

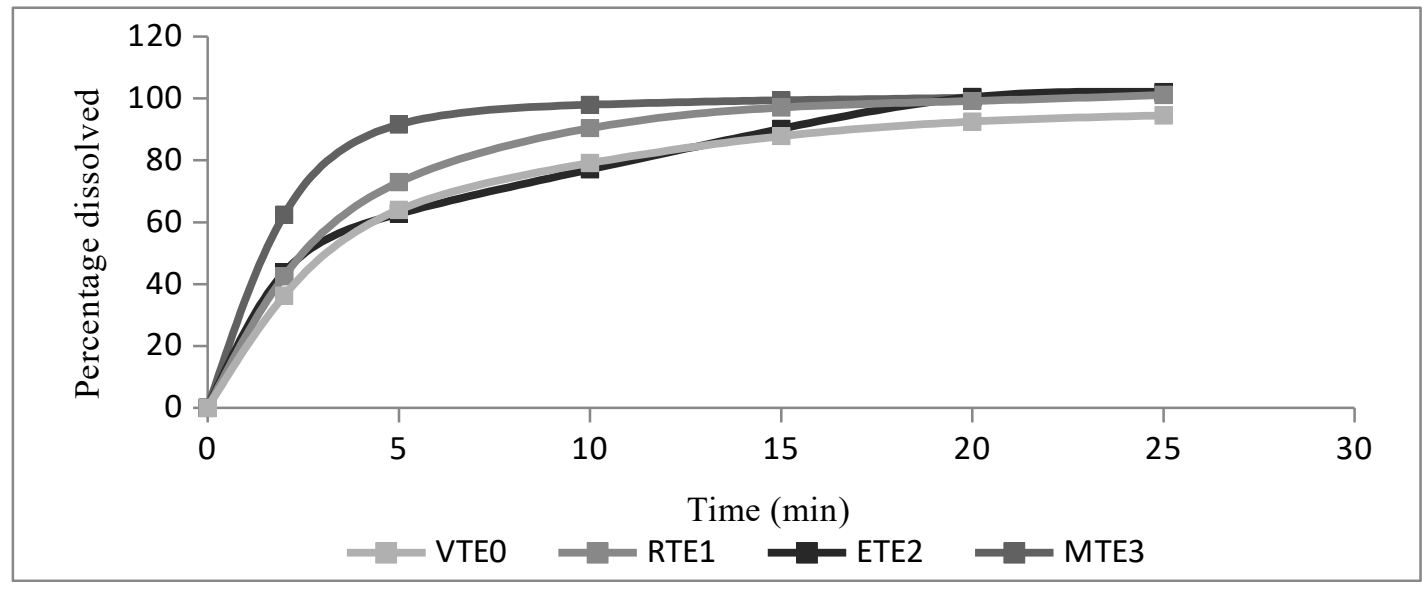

Figure 9. Graphic representation of the dissolution profile of tenoxicam at different pharmaceutical laboratories VTE0, RTE1, ETE2 and MTE3. 
Drug Anal Res, 2018; 02, 27-36

\section{Analysis of the tenoxicam tablets}

The mean results referring to the dissolution profile of the four pharmaceutical specialties are described in Table 3 and graphically represented in Figure 9. The DE\% obtained from the areas under the curves (AUC) between 0 and 50 minutes and the total area of the rectangle $\left(\mathrm{ASC}_{\mathrm{TR}}\right)$ for the active pharmaceutical ingredients of tenoxicam presented values of $60.30 \%$ for GTE1, $60.70 \%$ for STE2 and $72.34 \%$ for NTE3. The analysis of variance (ANOVA) showed that there was no significant statistical difference between the active pharmaceutical ingredients regarding dissolution efficiency $\left(\mathrm{F}_{\text {cal }}=5.40 ; \quad \mathrm{p}<0.01\right)$. The dissolution profiles of the active ingredients evaluated, GTE1, STE2 and NTE3 present similar dissolution profiles, possibly because they have the same crystalline structure (form III), with a similar crystallinity index.

Although the industrial processes may cause changes in the crystalline structure of pharmaceutical compounds, tenoxicam in different active ingredients presented the same crystalline structure (form III). Although different dissolution velocities occur between the active ingredients, their release profile was not compromised.

According to the data presented, the tenoxicam tablets evaluated had similar profiles to each other, releasing over $85 \%$ of the drug in 15 minutes.

Table 4 shows the values of $f 1$ and $f 2$ of the tenoxicam tablets VTE0, RTE1, ETE2 and MTE3. Considering that the values of $f 1$ should be between 0 and 15 and $f 2$ between 50 and $100,{ }^{(22)}$ the results indicate differences between the pharmaceutical specialties. The dissolution profiles of the finished products VTE0, RTE1 and ETE2 are similar, while product MTE3 is different from these, with $f 1$ values above what the specification $(23.95 ; 22.07)$ and $f 2$ values below it $(32.86 ; 42.36 ; 34.27)$.

Table 4. Values of $f 1$ and $f 2$ resulting from the comparisons between the tenoxicam tablets.

\begin{tabular}{|c|c|c|c|c|c|c|c|c|}
\hline & \multicolumn{2}{|c|}{ VTEO } & \multicolumn{2}{|c|}{ RTE1 } & \multicolumn{2}{|c|}{ ETE2 } & \multicolumn{2}{|c|}{ MTE3 } \\
\hline & $f 1$ & $f 2$ & $f 1$ & $f^{2}$ & $f 1$ & $f 2$ & $f 1$ & \\
\hline $\begin{array}{l}\text { VTE0 } \\
\end{array}$ & & & 11.81 & 51.88 & 4.82 & 68.59 & 23.95 & 32.86 \\
\hline RTE1 & 11.81 & 51.88 & & & 10.45 & 51.93 & 13.77 & 42.36 \\
\hline ETE2 & 4.82 & 68.59 & 10.45 & 51.93 & & & 22.07 & 34.27 \\
\hline MTE3 & 23.95 & 32.86 & 13.77 & 42.36 & 22.07 & 34.27 & & \\
\hline
\end{tabular}

When applying the dependent mathematical models it was found that the kinetics of tenoxicam release from the formulations followed zero order kinetics. The graphics generated by the zero order kinetics equation for the samples called VTE0, RTE1, ETE2 and MTE3 produced determination coefficients $\left(\mathrm{R}^{2}\right)$ superior to those calculated by the first order model. Besides, when evaluating the kinetic models tested, the o MSC (Model Selection Criteria) assigned higher values to the zero order model; Therefore the zero order model describe the tenoxicam release profile more appropriately, since the most appropriate model is the one which presents a value of $\mathrm{R}^{2}$ close to one (Table 5).
Table 5. Determination coefficients $\left(\mathrm{R}^{2}\right)$ and values of MSC obtained from the dissolution profiles of tablets containing tenoxicam, when applying zero order and first order kinetic equations.

\begin{tabular}{ccccc}
\hline \multirow{2}{*}{ Samples } & \multicolumn{4}{c}{ Kinetic Models } \\
\cline { 2 - 5 } & $\begin{array}{c}\text { Zero } \\
\text { Order }\end{array}$ & MSC & $\begin{array}{c}\text { First } \\
\text { Order }\end{array}$ & MSC \\
\hline VTE0 & 0.9044 & 1.0100 & 0.8516 & 0.5700 \\
RTE1 & 0.9150 & 1.1323 & 0.8653 & 0.6718 \\
ETE2 & 0.9461 & 1.5800 & 0.9129 & 1.1081 \\
MTE3 & 0.9920 & 0.1200 & 0.8513 & 0.0454 \\
\hline
\end{tabular}

Based on the zero order kinetic model, which was approached most closely by the release mechanism of tenoxicam, the release constant $(k)$, the half-life $\left(t_{50 \%}\right)$ and the amount released in 25 minutes $\left(\mathrm{Q}_{25}\right)$ were calculated. Table 6 shows that the specialties VTE0, RTE1, ETE2 and MTE3 presented very similar amounts of drug released in 25 minutes, and specialty MTE3 exhibited the shortest half-life among the formulations, indicating better product performance.

Dissolution efficient DE (\%) for the different tenoxicam tablets was also determined (Table 6). This parameter was obtained from the areas under the curves (AUC) between 0 and 25 minutes, and the total area of the rectangle $\left(\mathrm{ASC}_{\mathrm{TR}}\right)$. Among the pharmaceutical specialties of tenoxicam evaluated, the MTE3 presentation exhibited a DE\% value close to $100 \%$. Analysis of variance (ANOVA) showed that there is a significant statistical difference between the pharmaceutical specialties regarding the values of DE \% $\left(F_{c a l}=66.55 ; \mathrm{p}<0.01\right)$. According to the values obtained for the Tukey test, multiple comparison of means and $p<0.01$, it was found that the tenoxicam tablet MTE3 presented a significant statistical difference between the formulations evaluated, thus reflecting a difference in its dissolution profile in relation to the other pharmaceutical specialties.

Table 6. Release constant $(k)$, half life time $\left(t_{50 \%}\right)$ and amount released in 25 minutes $\left(\mathrm{Q}_{25}\right)$, according to the equation adjusted by the zero order model, of the tenoxicam tablets.

\begin{tabular}{ccccc}
\hline Samples & $\begin{array}{c}\boldsymbol{k} \\
\left(\mathbf{m i n}^{-\mathbf{1}}\right)\end{array}$ & $\begin{array}{c}\mathbf{T}_{\mathbf{5 0} \%} \\
(\mathbf{m i n})\end{array}$ & $\begin{array}{c}\mathbf{Q}_{\mathbf{2 5}} \\
\mathbf{( \% )}\end{array}$ & $\begin{array}{c}\mathbf{D E} \\
\mathbf{( \% )}\end{array}$ \\
\hline VTE0 & 0.00053 & 8.93 & 19.26 & 75.23 \\
RTE1 & 0.00054 & 8.08 & 20.52 & 83.69 \\
ETE2 & 0.00053 & 9.05 & 20.39 & 78.19 \\
MTE3 & 0.00057 & 7.71 & 20.91 & 90.63 \\
\hline
\end{tabular}

The tenoxicam tablets evaluated VTE0, RTE1, ETE2 and MTE3 presented qualitatively similar dissolution profiles, since the mathematical model for the best fit of the data was the same for all formulations. However, applying the kinetic model identified differences regarding the half life and dissolution velocity, especially for the commercial product MTE3. This result corroborated the data obtained by the independent model $f 1$ and $f 2$, which indicated a difference in the dissolution profile for this same pharmaceutical specialty.

Different methods used for comparison purposes can give rise to different results. This is because some parameters are more discriminating for differentiating pharmaceutical 
specialties. ${ }^{(23)}$ However, the results obtained by comparing the independent models $f 1$ and $f 2$, kinetic parameters, and values of dissolution efficiency, proved concordant with the medication MTE3.

Although the tenoxicam tablets have the same crystalline structure, they presented different DE\%. The pharmaceutical tablets VTE0, RTE1 e ETE2 presented a slower dissolution and a smaller $\mathrm{DE} \%$, possibly due to the higher crystallinity index present in their chemical structures. The faster dissolution and higher DE\% of the pharmaceutical product MTE3 may possibly be the result of the lower crystallinity index. The substances with a lower crystallinity index, are mostly more soluble than materials with a higher crystallinity index, ${ }^{(24)}$ which fact has possibly influenced the results expounded above.

\section{Conclusions}

Polymorphism affects physical, chemical and biopharmaceutical properties, both for active ingredients and for pharmaceutical formulations, and it is a relevant physicochemical parameter for the pharmaceutical industry. The analyses performed showed that the active pharmaceutical ingredients and the pharmaceutical tablets available in the market, when submitted to analysis by XRD presented similar crystalline structures (form III). Although the pharmaceutical formulations present different dissolution profiles, the industrial process to which tenoxicam was submitted did not have a significant impact on its crystalline structure, considering that the quality of the pharmaceutical products was maintained.

\section{References}

1. Cuffini SL, Pitaluga AJ, Tombari D. Polimorfismo em fármacos. In: Storpirtis S, Gonçalves JE, Chiann C, Gai MN. Biofarmacotécnica. Rio de Janeiro: Guanabara Koogan; 2011:21-30.

2. LU J, Rohani S. Polymorphism and crystallization of active pharmaceutical ingredients (APIs). Curr. Med. Chem. 2009;16:884-905.

3. Allen LVJ, Popovich NG, Ansel HC. Ansel's Pharmaceutical Dosage Forms and Drug Delivery Systems. 9th ed. Baltimore: Lippincott Willians \& Willians; 2011.

4. Singhal D, Curatolo W. Adv. Drug polymorphism and dosage form design: a practical perspective. Drug Delivery Rev 2004;56:335-347.

5. Capucho HC, Mastroianni PC, Cuffini S. Farmacovigilância no Brasil: a relação entre polimorfismo de fármacos, efetividade e segurança dos medicamentos. Rev. Ciênc. Farm. Básica Apl. 2008;29:277-283.
6. Oliveira MA, Yoshida MI, Gomes ECL. Análise térmica aplicada a fármacos e formulações farmacêuticas na indústria farmacêutica. Quim. Nova 2011;34:1224-1230.

7. Morris KR, Griesser UJ, Eckhardt CJ, Stowel JG. Theoretical approaches to physical transformations of active pharmaceutical ingredients during manufacturing processes. Adv. Drug Deliver. Rev. 2001;48:91-114.

8. Stephenson GA. Applications of X-Ray Powder Diffraction in the Pharmaceutical Industry. Rigaku J. 2005;22:2-15.

9. Florence AT, Attwood D. Physicochemical Principles of Pharmacy. 3th ed. London: Palgrave Macmillan; 1998.

10. Zhang J, Tan X, Gao J, Fan W, Gao Y, Qian S. Characterization of two polymorphs of lornoxicam. Journal of Pharmacy and Pharmacology. J. Pharm. Pharmacol. 2013;65:44-52.

11. Cantera RG, Leza MG, Bachiller CM. Solid Phases of Tenoxicam. J. Pharm. Sci. 2002;91:2240-2251.

12. BRITISH PHARMACOPOEIA COMMISSION. British Pharmacopoeia 2013. London: The Stationery Office, 2013.

13. Alladi S, Shastri NR. Semi solid matrix formulations of meloxicam and tenoxicam: an in vitro and in vivo evaluation. Arch. Pharmacal Res. [Online] 2014. Apr. Available from: http://link.springer.com/article/10.1007/ s12272-014-0396-3/fulltext.html. [last acess 10 Jan 2016].

14. Kim T, Kang E, Chun I, Gwak H. Pharmacokinetics of formulated tenoxicam transdermal delivery systems. J. Pharm. Pharmacol. 2008;60:135-138.

15. Yu L, Reutzel-Edens SM, Mitchell CA. Crystallization and polymorphism of conformationally flexible molecules: Problems, patterns, and strategies. Org. Process Res. Dev. 2000;4:396-402.

16. Schmidt AC, Schwarz I, Mereiter K. Polymorphism and Pseudopolymorphism of salicaine and salicaine hydrochloride crystal polymorphism of local anaesthetic drugs. J. Pharm. Sci. 2006;95:1097-1113.

17. Patel JR, Carlton RA, Needham TE, Chichester CO, Vogt FG. Preparation, structural analysis, and properties of tenoxicam cocrystals. International Journal of Pharmaceutics. Int. J. Pharm. 2012;436:685-706.

18. Bolla G, Sanphui P, Nangia A. Solubility advantage of tenoxicam phenolic cocrystals compared to salts. Cryst. Growth Des. 2013;13:1988-2003.

19. Lin SY. An overview of famotidine polimorphs: solidstate characteristics, termodynamics, polymorphic 
transformation and quality control. Pharm Res 2014;31:1619-1631.

20. Karabas I, Orkoula MG, Kontoyannis CG. Analysis and stability of polymorphs in tablets: the case of Risperidone. Talanta 2007;71:1382-1386.

21. Pavia D, Kriz G, Lampman G. Introduction to spectroscopy: A guide for students of organic chemistry. 3rd ed. Washington: Brooks/Cole Thomson Learning; 2001.

22. Moore JW, Flanner HH. Mathematical comparison of dissolution profiles. Pharm. Technol. 1996;20:64-74.

23. Yuksel N, Kanik AE, Baykara T. Comparison of in vitro dissolution profiles by ANOVA based, model dependent and independent methods. Int. J. Pharm 2000;209:57-67.

24. Gibaldi M. Biopharmaceutics and Clinical Pharmacokinetics. 4th ed. Philadelpia: Lea \& Febiger, 1991. 